\title{
Heteroclinic optimal control solutions for attitude motion planning
}

\author{
James D. Biggs*, Craig Maclean, Albert Caubet
}

\begin{abstract}
An analytical attitude motion planning method is presented that exploits the heteroclinic connections of an optimal kinematic control problem. This class of motion, of hyperbolic type, supply a special case of analytically defined rotations that can be further optimised to select a suitable reference motion that minimises accumulated torque and the final orientation error amongst these motions. This analytical approach could be used to improve the overall performance of a spacecraft's attitude dynamics and control system when used alongside current flight tested tracking controllers. The resulting algorithm only involves optimising a small number of parameters of standard functions and is simple to implement.

Keywords: optimal control, Heteroclinic connections, motion planning.
\end{abstract}

\section{INTRODUCTION}

This work is motivated by the problem of precisely controlling nano-spacecraft, such as the UKube 1, ESA's OPS-SAT or STRaND-1, from an initial orientation to a prescribed final orientation. The attitude control of nanospacecraft is usually undertaken using magnetorquers for detumbling. However, there is a desire both from a commercial and scientific point of view to perform large slew motions and fine pointing of nano-spacecraft. Recent developments in nano spacecraft reaction wheels could enable such maneuvers. However, the maximum torque capability of current nano-spacecraft reaction wheels is, for example, $6.25 \times 10^{-4}$ $\mathrm{Nm}$ (MAI-400) or $1 \times 10^{-3} \mathrm{Nm}$ (RW-0.007-4) compared to communications satellites of $10 \mathrm{Nm}$ to $70 \mathrm{Nm}$. Furthermore, the capability of nano-spacecraft on-board processors ranges from around $30 \mathrm{MHz}$ on the UKube 1 to potentially 600 $\mathrm{MHz}$ on the OPS-SAT. Therefore, the limited torque and small available processing power pose key challenges.

It is therefore important to consider a number of practical objectives when designing attitude maneuvers for nanospacecraft; to minimise the final pointing error, to minimise the accumulated torque expended during a maneuver and to minimise the computational cost of the motion planning and control algorithm. As is now shown, it is straightforward to formulate a multi-objective optimal control problem that minimises torque during a maneuver while matching the final desired orientation at some final time $T$. However, the additional requirement of low computation has to be understood through implementation and simulation.

The attitude dynamics of a spacecraft [1] are:

$$
\begin{aligned}
& \dot{\omega}_{1}=\lambda_{1} \omega_{2} \omega_{3}+\frac{T_{1}}{I_{1}} \\
& \dot{\omega}_{2}=\lambda_{2} \omega_{1} \omega_{3}+\frac{T_{2}}{I_{2}} \\
& \dot{\omega}_{3}=\lambda_{3} \omega_{1} \omega_{2}+\frac{T_{3}}{I_{3}}
\end{aligned}
$$

where $\omega_{1}, \omega_{2}, \omega_{3}$ are the components of angular velocity $I_{1}, I_{2}, I_{3}$ are the principal moments of inertia and $\lambda_{1}=\left(I_{2}-\right.$
$\left.I_{3}\right) / I_{1}, \lambda_{2}=\left(I_{3}-I_{1}\right) / I_{2}$ and $\lambda_{3}=\left(I_{1}-I_{2}\right) / I_{3}$ and where $T_{1}, T_{2}, T_{3}$ define the components of the torque vector $\mathbf{u}=$ $\left[T_{1}, T_{2}, T_{3}\right]^{T}$ in the absence of perturbations. The orientation of a spacecraft is globally defined by the rotation matrix $R(t) \in S O(3)$ where $S O(3)$ is the Special Orthogonal Group that satisfies the kinematic relationship:

$$
\dot{R}(t)=R(t) \Omega
$$

where $\Omega=\omega_{1} A_{1}+\omega_{2} A_{2}+\omega_{3} A_{3}$ where $A_{1}, A_{2}, A_{3}$ are the basis of the Lie algebra $\mathfrak{s o}(3)$ of the Lie Group $S O(3)$ :

$$
\begin{aligned}
A_{1} & =\left(\begin{array}{ccc}
0 & 0 & 1 \\
0 & 0 & 0 \\
-1 & 0 & 0
\end{array}\right), \quad A_{2}=\left(\begin{array}{ccc}
0 & 0 & 0 \\
0 & 0 & -1 \\
0 & 1 & 0
\end{array}\right), \\
A_{3} & =\left(\begin{array}{ccc}
0 & -1 & 0 \\
1 & 0 & 0 \\
0 & 0 & 0
\end{array}\right) .
\end{aligned}
$$

where physically $A_{1}, A_{2}, A_{3}$ define the infinitesimal rotations in the roll, pitch and yaw directions respectively with the Lie Bracket defined by $[\cdot, \cdot]=X Y-Y X$ where $\left[A_{1}, A_{2}\right]=$ $-A_{3},\left[A_{1}, A_{3}\right]=A_{2},\left[A_{2}, A_{3}\right]=-A_{1}$. We choose to define our motion planning problem on $S O(3)$ as it defines rotations uniquely and globally and thus will avoid any problem with potential unwinding that can occur with a quaternion representation [2].

For such a problem we wish to minimise the accumulated torque subject to the boundary conditions $R_{0}=R(0)$ and $R_{T}=R(T)$ where $T$ is the final time (the final time can be fixed or free). To minimise the final orientation error it is natural to choose a metric related to the geometry of the Lie Group $S O(3)$ that describes the minimum distance error function at the final time $T$ such as $\left\|R_{e}\right\|=\left\langle\log R_{e}, \quad \log R_{e}\right\rangle^{1 / 2}$ where $\langle A, B\rangle=-\frac{1}{2} \operatorname{trace}(A B)$ where $A, B \in \mathfrak{s o}(3)$ and $R_{e}=$ $R_{d}{ }^{-1} R(T)$ where $R_{d}$ is the desired orientation at the final time $T$. This particular metric ensures that the error between the actual and desired motion is measured along a geodesic on $S O(3)$. In addition we require that during the maneuver the accumulated torque, $\mathbf{u}_{a}=\int_{0}^{T}\|\mathbf{u}(t)\|_{\mathbb{R}^{3}} d t$, is minimised with $\|\mathbf{u}(t)\|_{\mathbb{R}^{3}}=\langle\cdot, \cdot\rangle_{\mathbb{R}^{3}}^{1 / 2}$ and where $\langle\cdot, \cdot\rangle_{\mathbb{R}^{3}}$ is the usual dot product. Then we can state a full orientation motion planning problem given final time $T$ and $R_{0}=R(0)$ as one of minimising the function:

$$
J_{1}=\left\|R_{e}\right\|^{2}+\alpha \mathbf{u}_{a}
$$

with respect to the differential constraints (1) and (2) where $\alpha$ is a constant weight. Alternatively if one is only concerned with the pointing position $x(T)=R(T) e_{1}$ of the spacecraft 
then given $x_{0}=R(0) e_{1}$ we can define a reduced motion planning problem that minimises the function:

$$
J_{2}=\left\|\left(R(T)-R_{d}\right) e_{1}\right\|_{\mathbb{R}^{3}}+\alpha \mathbf{u}_{a}
$$

where $e_{1}=\left[\begin{array}{lll}1 & 0 & 0\end{array}\right]^{T}$ with respect to the differential constraints (1) and (2).

Such a problem could be solved using numerical optimisation software such as pseudo-spectral methods which have been used to design optimal attitude motions for the International Space Station [3]. However, such methods are computationally expensive for the available processing power on-board nano-spacecraft. Even in the case that it was possible to use such a numerical optimiser to minimise this cost function on-board it is still desirable to minimise the computational requirement to "free up" processing power to perform essential operating tasks. In this paper we aim to strike a balance between minimising the cost function (4) (or (5)) and minimising the computational cost of doing so. The long term aim of this research is to develop an analytical method for motion planning that improves the efficiency of conventional controllers by tracking a designed motion rather than just a final desired orientation with low computational cost.

Analytically defined cost functions (as opposed to a cost function defined by an integral) are potentially favourable as to minimise them only requires the optimisation of a small number of parameters. In [4] pseudo-spectral methods were used to numerically generate a large number of optimal attitude motions of nano-spacecraft and then polynomial functions fit to these to compute the most appropriate form for use as analytical attitude planners. The key objective for polynomials is to find the most suitable order that accurately generates a near optimal motion while minimising computational expense. The paper [4] used a numerical optimisation to inform the design of an analytical motion planner. In this paper we take a different approach by exploiting the geometry of an optimal kinematic control problem to generate analytic curves of hyperbolic type. By considering a subset of feasible motions, of hyperbolic type, it is possible to formulate the cost function (4) (or (5)) analytically. This means that the original functional optimisation problem reduces to a much simpler parameter optimisation problem.

Previous work in this area has considered motions that correspond to analytic curves of trigonometric type [7]. Although these can define adequate motions that enhance conventional proportional controllers the planned motions are restricted to using periodic or arcs of periodic motion [5], [7]. In contrast analytically defined motions have been of elliptic type with Jacobi elliptic functions and an elliptic integral defining the motion [6]. This work showed that using quaternion feedback control the generated motions could be tracked using nano-reaction wheels. However, the presence of the elliptic integral meant that the computational expense increased dramatically relative to cases where the motion is described by elementary functions e.g. trigonometric [7], polynomial [4] or hyperbolic.
In summary the steps presented in this paper are outlined as follows:

1) Prune all possible feasible motions by formally defining an optimal control problem and assigning a general quadratic cost function of angular velocities.

2) Derive the analytic form for this general class of motion.

3) Identify the motions that correspond to the heteroclinic connections of the extremal curves (motions of hyperbolic type).

4) Using a process of inverse dynamics calculate an analytic expression for the accumulated torque.

5) Define an analytic multi-objective cost function of the accumulated torque and final pointing error at final time $T$.

6) Subject to prescribed boundary conditions optimise the parameters to minimise the cost function and define the appropriate attitude motion.

We begin by defining an optimal kinematic control problem on a Lie group [8].

\section{BACKGROUND ON OPTIMAL KINEMATIC CONTROL}

The first step in the procedure is to prune all possible feasible motions by defining a kinematic optimal control problem. The class of pruned kinematic motions that we consider are those that minimise the cost function:

$$
C=\frac{1}{2} \int_{0}^{T} c_{1} \omega_{1}^{2}+c_{2} \omega_{2}^{2}+c_{3} \omega_{3}^{2} d t
$$

subject to the kinematic constraint (2) between an initial orientation $R(0)$ and a final prescribed orientation $R(T)$. This cost function was originally proposed in [9] in order to keep the overall angular velocity low during the maneuver time $T$ avoiding high spin rates that can cause a spacecraft to start tumbling and to ensure that good tracking data can be obtained from the sensors. Such a cost function may also be desirable for flexible structures where slow maneuvers would avoid exciting the structural modes. We remind the reader that this is not a cost function that reflects the ultimate practical goals of our problem but defines a broad class of continuous motions that can be defined analytically and further optimised. To view this as a broad class of reference motions notice that when $c_{1}=I_{1}, c_{2}=I_{2}, c_{3}=I_{3}$ the cost function is equivalent to minimising the integral of the Lagrangian or kinetic energy of a rigid body. Thus, this class of motion includes the natural motions of a rigid body [7], [6]. In this case the optimal control problem is equivalent to a statement of the Principle of Least Action in mechanics.

An application of Pontryagin's Maximum Principle of optimal control to this problem is detailed in [9] and [10] and leads to the following optimal controls $\omega_{1}^{*}=M_{1} / c_{1}, \omega_{2}^{*}=$ $M_{2} / c_{2}, \omega_{3}^{*}=M_{3} / c_{3}$ where $M_{i}$ are the extremal curves which satisfy the following necessary conditions for optimality:

$$
\begin{aligned}
& \dot{M}_{1}=\frac{c_{2}-c_{3}}{c_{2} c_{3}} M_{2} M_{3} \\
& \dot{M}_{2}=\frac{c_{3}-c_{1}}{c_{1} c_{3}} M_{1} M_{3} \\
& \dot{M}_{3}=\frac{c_{1}-c_{2}}{c_{1} c_{2}} M_{1} M_{2}
\end{aligned}
$$


Note that when the constant weights $c_{1}, c_{2}, c_{3}$ of the cost function (5) coincide with the Principal moments of inertia $I_{1}, I_{2}, I_{3}$ of the spacecraft then (7) is simply the Euler equations where the extremal curves $M_{1}, M_{2}, M_{3}$ are the components of angular momentum. However, as in general $c_{1} \neq I_{1}, c_{2} \neq I_{2}, c_{3} \neq I_{3}$ the extremal curves define a broader class of smooth motions. It is well known that these equations are integrable with two conserved quantities [8]:

$$
H=\frac{1}{2}\left(\frac{M_{1}^{2}}{c_{1}}+\frac{M_{2}^{2}}{c_{2}}+\frac{M_{3}^{2}}{c_{3}}\right), \quad M=\left(M_{1}^{2}+M_{2}^{2}+M_{3}^{2}\right)
$$

which can be solved in terms of trigonometric functions when two of the weights of the cost function are equal [10]. In the general case these can be solved in terms of elliptic functions. Let

$$
\lambda_{1}=\left(\frac{c_{2}-c_{3}}{c_{2} c_{3}}\right)^{2}, \lambda_{2}=\left(\frac{c_{3}-c_{1}}{c_{1} c_{3}}\right)^{2}, \lambda_{3}=\left(\frac{c_{1}-c_{2}}{c_{1} c_{2}}\right)^{2},
$$

and using the conserved quantities we can write:

$$
\begin{array}{ll}
M_{2}^{2}=\alpha_{1} M_{1}^{2}-\beta_{1}, & M_{3}^{2}=k_{1} M_{1}^{2}-d_{1} \\
M_{1}^{2}=\alpha_{2} M_{2}^{2}-\beta_{2}, & M_{3}^{2}=k_{2} M_{2}^{2}-d_{2} \\
M_{1}^{2}=\alpha_{3} M_{3}^{2}-\beta_{3}, & M_{2}^{2}=k_{3} M_{3}^{2}-d_{3}
\end{array}
$$

where

$$
\begin{array}{ll}
\alpha_{1}=\frac{c_{2}\left(c_{1}-c_{3}\right)}{c_{1}\left(c_{3}-c_{2}\right)}, & \beta_{1}=\frac{c_{2}\left(2 c_{3} H-M\right)}{c_{2}-c_{3}} \\
k_{1}=\frac{c_{3}\left(c_{1}-c_{2}\right)}{c_{1}\left(c_{2}-c_{3}\right)}, & d_{1}=\frac{c_{3}\left(2 c_{2} H-M\right)}{c_{3}-c_{2}} \\
\alpha_{2}=\frac{c_{1}\left(c_{1}-c_{3}\right)}{c_{2}\left(c_{3}-c_{1}\right)}, & \beta_{2}=\frac{c_{1}\left(2 c_{3} H-M\right)}{c_{3}-c_{3}} \\
k_{2}=\frac{c_{3}\left(c_{2}-c_{1}\right)}{c_{2}\left(c_{1}-c_{3}\right)}, & d_{2}=\frac{c_{3}\left(2 c_{1} H-M\right)}{c_{3}-c_{1}} \\
\alpha_{3}=\frac{c_{1}\left(c_{3}-c_{2}\right)}{c_{3}\left(c_{2}-c_{1}\right)}, & \beta_{3}=\frac{c_{1}\left(2 c_{2} H-M\right)}{c_{1}-c_{2}} \\
k_{3}=\frac{c_{2}\left(c_{3}-c_{1}\right)}{c_{3}\left(c_{1}-c_{2}\right)}, & d_{3}=\frac{c_{2}\left(2 c_{1} H-M\right)}{c_{2}-c_{1}}
\end{array}
$$

The optimal solutions can then be expressed in the quadratic form:

$$
\left(\dot{M}_{i}\right)^{2}=\lambda_{i}\left(\alpha_{i} M_{i}^{2}-\beta_{i}\right)\left(k_{i} M_{i}^{2}-d_{i}\right)
$$

where $i=1,2,3$ and $\lambda_{i}$ are defined in (9). The solution of (12) is then a Jacobi elliptic function where explicitly we have:

$$
M_{i}=\sqrt{b_{i} / a_{i}} \sin \left(z_{i}\right)
$$

where $a_{i}=\lambda_{i} \alpha_{i}$ and $b_{i}=\lambda_{i} \beta_{i}$ and:

$$
z_{i}=\operatorname{am}\left( \pm \sqrt{a_{i} d_{i}} t+\beta_{i}, \frac{b_{i} k_{i}}{a_{i} d_{i}}\right)
$$

where $\operatorname{am}(\cdot, \cdot)$ is the Jacobi amplitude function [12] and the constant $\beta_{i}=\sin ^{-1}\left(\operatorname{am}\left(\frac{\sqrt{a_{i}} M_{i}(0)}{\sqrt{b_{i}}}, \frac{b_{i} k_{i}}{a_{i} d_{i}}\right)\right)$.

These solutions can then be integrated to obtain the rotation matrix corresponding to these optimal controls. The corresponding rotations are expressed in terms of Jacobi elliptic functions and an elliptic integral of the third kind, see [11] for the general integration procedure and for the analogous integration for a free rigid body motion on $S U(2)$ [6]. As one of the goals of this approach is to reduce the computational complexity of the on-board motion planning method the elliptic integrals involved in defining the rotations are not ideal. In fact the original suggestion of Spindler [9] was to use a numerical shooting method to solve the necessary conditions for optimality (7) while matching the boundary conditions subject to the kinematic constraint (2). An analytical method that involves the evaluation of elliptic integrals would likely not offer a vast improvement in computational expense in comparison to this numerical approach. In this paper we set out a method based on the heteroclinic connections of the general optimal control solutions.This reduces the amount of available feasible solutions to be further optimised using the cost function (4) but reduces the computation time considerably as it only involves standard functions. Moreover, by defining the rotation matrix $R(t)$ in terms of the local coordinates $\phi_{1}, \phi_{2}, \phi_{3}$ :

$$
R(t)=\exp \left(\phi_{1} A_{3}\right) \exp \left(\phi_{2} A_{2}\right) \exp \left(\phi_{3} A_{3}\right)
$$

following the procedure in [10] to solve the general optimal kinematic control problem of minimising (6) subject to the kinematic constraint (2) it can be shown that an elliptic integral appears as the solution to the coordinate $\phi_{1}$ which can be expressed as an integral function of the extremal curve $M_{3}$ :

$$
\phi_{1}=\int \frac{\sqrt{M}\left(2 H-M_{3}^{2} / c_{3}\right)}{M-M_{3}^{2}} d t
$$

where in the general case $M_{3}$ is defined as the Jacobi elliptic function (13) which yields an explicit expression of the form:

$$
\begin{aligned}
& \phi_{1}=\int \frac{a-b s n^{2}(c t+d, m)}{e-f s n^{2}(c t+d, m)} d t=\frac{b}{d} t+ \\
& \left(\frac{a f-b e}{c e}\right) \Pi\left[\frac{f}{e}, a m(c t+d), m\right]+\beta
\end{aligned}
$$

In (17) $\Pi$ is the elliptic integral of the third kind [12], $a, b, c, d, e, f, m$ are constants related to $M_{1}(0), M_{2}(0), M_{3}(0), c_{1}, c_{2}, c_{3}$ and $\beta$ is a constant of integration. This form is not particularly convenient for motion planning as the evaluation of the elliptic integral is computationally more expensive than those consisting of standard functions.

To avoid the calculation of an elliptic integral one can consider the particular case when $M_{3}$ degenerates from a Jacobi elliptic function to a hyperbolic tan function (at $m=$ 1). This corresponds to a heteroclinic connection in the phase space of the extremal curves analogous to the heteroclinic connection of a free rigid body in the Euler equations which connects two unstable saddle equilibrium points. In this case, as is shown later, $\phi_{1}=\frac{2 H}{\sqrt{M}} t+\beta$. By considering the heteroclinic solutions of the kinematic control problem we avoid the necessity of evaluating elliptic integrals. This paper will proceed to outline a method for attitude motion planning based on these special solutions.

\section{HETEROCLINIC CONNECTIONS}

The heteroclinic connections of the necessary conditions for optimality (7) are defined when $\frac{a_{i} d_{i}}{b_{i} k_{i}}=1$ in (14). We look at the case when $M_{3}$ degenerates to a hyperbolic function when $\frac{a_{3} d_{3}}{b_{3} k_{3}}=1$ and it is straightforward to show that this 
occurs when the weight of the cost function $c_{3}=\frac{M}{2 H}$. For this choice of $c_{3}$ the Hamiltonian (8) can be expressed as:

$$
H=\frac{1}{2}\left(\frac{M_{1}^{2}}{c_{1}}+\frac{M_{2}^{2}}{c_{2}}+\frac{M_{3}^{2}\left(M_{1}^{2} / c_{1}+M_{2}^{2} / c_{2}\right)}{M_{1}^{2}+M_{2}^{2}}\right)
$$

However, we cannot set more than one weight to this value as it would mean weights are equal and would revert to the symmetric case with either periodic or constant angular velocities. Setting $c_{3}=\frac{M}{2 H}$ in (13) yields:

$$
M_{3}=s_{3} \sqrt{M} \tanh (\gamma t+C)
$$

then using the relationships (10) we can obtain $M_{1}$ and $M_{2}$ :

$$
M_{1}=A \operatorname{sech}(\gamma t+C), \quad M_{2}=B \operatorname{sech}(\gamma t+C)
$$

where $\quad \gamma=\sqrt{\frac{\left(M-2 c_{1} H\right)\left(2 c_{2} H-M\right)}{c_{1} c_{2} M}}, \quad A=s_{1} \sqrt{\frac{c_{1}\left(2 H c_{2}-M\right)}{c_{2}-c_{1}}}$, $B=s_{2} \sqrt{\frac{c_{2}\left(M-2 H c_{1}\right)}{c_{2}-c_{1}}}$ and $C=\operatorname{sech}^{-1}\left(s_{1} \sqrt{\frac{c_{2}-c_{1}}{c_{1}\left(2 H c_{2}-M\right)}} M_{1}(0)\right)$ where $s_{1}, s_{2}, s_{3}$ are either \pm 1 and define the four heteroclinic connections in the phase space of the extremal curves. Each connection is defined uniquely in Table 1:

\begin{tabular}{|l|l|l|l|}
\hline Seperatrix & $s_{1}$ & $s_{2}$ & $s_{3}$ \\
\hline 1 & +1 & +1 & +1 \\
\hline 2 & -1 & -1 & +1 \\
\hline 3 & -1 & +1 & -1 \\
\hline 4 & +1 & -1 & -1 \\
\hline
\end{tabular}

Note that these combinations of signs are equivalent to $s_{1} s_{2}=$ $s_{3}$. The equations (20) and (19) can then be used along with the equations (1) to determine the ideal torque magnitudes required to perform these motions $\|\mathbf{u}\|_{\mathbb{R}^{3}}=\sqrt{T_{1}^{2}+T_{2}^{2}+T_{3}^{2}}$ in terms of the extremal curves where

$$
\begin{aligned}
& T_{1}=\frac{I_{1}}{c_{1}} \dot{M}_{1}-\frac{\left(I_{2}-I_{3}\right) 2 H}{I_{1} c_{2} M} M_{2} M_{3} \\
& T_{2}=\frac{I_{2}}{c_{2}} \dot{M}_{2}-\frac{\left(I_{3}-I_{1}\right) 2 H}{I_{2} c_{1} M} M_{1} M_{3} \\
& T_{3}=\frac{2 H I_{3}}{M} \dot{M}_{3}-\frac{\left(I_{1}-I_{2}\right)}{I_{3} c_{1} c_{2}} M_{1} M_{2}
\end{aligned}
$$

Substituting in the values (19) and (20) and their derivatives into (21) we can calculate $\mathbf{u}_{a}$, part of the cost function (4), analytically in terms of hyperbolic functions of time and the constants $c_{1}, c_{2}, M_{1}(0), M_{2}(0), M_{3}(0)$. It is then a matter of optimising $c_{1}, c_{2}, M_{1}(0), M_{2}(0), M_{3}(0)$ to minimise $\mathbf{u}_{a}$ for a given $T, R(0)$ and desired final orientation $R(T)$. However, it is necessary to include the final orientation error in the cost function and we proceed to compute this motion analytically.

\section{CORRESPONDING ROTATION MATRIX OF HYPERBOLIC TYPE}

The procedure for integrating these equations are detailed in a number of papers [6], [8], [11]. Here we briefly explain the procedure which exploits the Lax pair structure to determine the heteroclinic connection. The extremals (7) can be expressed in vector form $\dot{L}=\nabla H \times L$ where $L=\nabla M, \nabla H \in$ $\mathbb{R}^{3}$ where $\nabla$ is the gradient or in Lax Pair form on the Lie group $\mathrm{SO}(3) \dot{L}=[L, \nabla H]$ where $L=\nabla M, \nabla H \in \mathfrak{s o}(3)$. This form is often convenient for integrating the system to obtain the rotation matrix $R(t)$ by exploiting the general solution of the Lax Pair:

$$
L(t)=R(t)^{-1} L(0) R(t)
$$

which can be conjugated to:

$$
R(t) L(t) R(t)^{-1}=\sqrt{M} A_{3}
$$

then substituting (15) into (23) and (2) the following expressions can be obtained through algebraic manipulation:

$$
\begin{aligned}
& \cos \phi_{2}=\frac{M_{3}}{\sqrt{M}}, \sin \phi_{2}=\frac{\sqrt{M-M_{3}^{2}}}{\sqrt{M}} \\
& \cos \phi_{3}=\frac{M_{1}}{\sqrt{M-M_{3}^{2}}}, \sin \phi_{3}=\frac{M_{2}}{\sqrt{M-M_{3}^{2}}}
\end{aligned}
$$

which for the extremal curves of hyperbolic type $(19,20)$ is explicitly:

$$
\begin{aligned}
& \cos \phi_{2}=s_{1} s_{2} \tanh (\gamma t+C), \quad \sin \phi_{2}=\operatorname{sech}(\gamma t+C) \\
& \cos \phi_{3}=\frac{A}{\sqrt{M}}, \quad \sin \phi_{3}=\frac{B}{\sqrt{M}}
\end{aligned}
$$

then substituting (15) into (2) and using the expressions (25) leads to the integral (16) and with $c_{3}=M / 2 H$ simplifies to:

$$
\phi_{1}=\frac{2 H}{\sqrt{M}} t+\beta
$$

where $\beta$ is a constant of integration. Then substituting (26) and (25) into (15) yields the rotation matrix. Then the general solution can be expressed as:

$$
R_{\text {gen }}(t)=R_{\text {gen }}(0) R(0)^{-1} R(t)
$$

where $R(t)=\left(\begin{array}{lll}x & y & z\end{array}\right)$ where the orthonormal vectors $x, y, z$ are defined by:

$$
\begin{gathered}
x=\frac{1}{\sqrt{M}}\left[\begin{array}{c}
A \cos \phi_{1}-B s_{1} s_{2} \tanh (\gamma t+C) \sin \phi_{1} \\
A \sin \phi_{1}+B s_{1} s_{2} \tanh (\gamma t+C) \cos \phi_{1} \\
B \operatorname{sech}(\gamma t+C)
\end{array}\right] \\
y=\frac{1}{\sqrt{M}}\left[\begin{array}{c}
-A s_{1} s_{2} \tanh (\gamma t+C) \sin \phi_{1}-B \cos \phi_{1} \\
A s_{1} s_{2} \tanh (\gamma t+C) \cos \phi_{1}-B \sin \phi_{1} \\
A \operatorname{sech}(\gamma t+C)
\end{array}\right] \\
z=\left[\begin{array}{c}
\sin \phi_{1} \operatorname{sech}(\gamma t+C) \\
-\cos \phi_{1} \operatorname{sech}(\gamma t+C) \\
s_{1} s_{2} \tanh (\gamma t+C)
\end{array}\right]
\end{gathered}
$$

From this analytic solution we can then define an appropriate error function of the final orientation. A suitable error function on the rotation groups $S O(3)$ can be defined as $\left\|R_{e}(T)\right\|=\left\langle\log R_{e}(T), \quad \log R_{e}(T)\right\rangle^{1 / 2}$. Given some final desired orientation $R_{d}$ we calculate $R_{e}=R_{d}^{T} R_{g e n}(T)$ and compute the $\log$ of this function using the equation [13] $\log R_{e}=\frac{\sin ^{-1}\|y\|}{\|y\|} \hat{y}$ where

$$
\hat{y}=\frac{1}{2}\left(R_{e}-R_{e}^{T}\right)
$$

where $\hat{\cdot}$ defines the map between a vector in $\mathbb{R}^{3}$ and the Lie algebra $\mathfrak{s o}(3)$ defined by:

$$
\begin{aligned}
& \hat{\imath}: \mathbb{R}^{3} \rightarrow \mathfrak{s o}(3) \\
& {\left[\begin{array}{l}
x_{1} \\
x_{2} \\
x_{3}
\end{array}\right] \mapsto\left(\begin{array}{ccc}
0 & -x_{3} & x_{2} \\
x_{3} & 0 & -x_{1} \\
-x_{2} & x_{1} & 0
\end{array}\right)}
\end{aligned}
$$


Therefore we can compute the original cost function (4) amongst all heteroclinic connections of motions that minimise the cost function (5). We note that this analytically defined cost function is defined in terms of five independent parameters $M_{1}(0), M_{2}(0), M_{3}(0), c_{1}, c_{2}$ which can be optimised to minimise $\left\|R_{e}(T)\right\|$. A simpler pointing problem can be defined by minimising $\left\|x-x_{d}\right\|_{\mathbb{R}^{3}}$. In the following section we present an example for the reduced attitude motion planning problem.

\section{Motion PLANNING ALGORITHM}

In this final section we discuss a few practicalities of implementation and illustrate this with a simple example of re-pointing a spacecraft from an intial pointing direction $x(0)=\left[\begin{array}{lll}1 & 0 & 0\end{array}\right]^{T}$ to a desired final pointing direction of $x_{d}=\left[\begin{array}{lll}0 & 0.22942 & 0.97333\end{array}\right]^{T}$ in a fixed time of 100 seconds. We assume the principal moments of inertia of the nano-spacecraft UKube 1 with $I_{1}=$ $0.0109 \mathrm{kgm}^{2}, I_{2}=0.0504 \mathrm{kgm}^{2}, I_{3}=0.0506 \mathrm{kgm}^{2}$. In order to plan a suitable motion we need to optimize the parameters $M_{1}(0), M_{2}(0), M_{3}(0), c_{1}>0, c_{2}>0$ to minimize the function (5). Therefore, it is necessary to state the conserved quantities $H, M$ in terms of these parameters:

$$
\begin{aligned}
& H=\frac{1}{2}\left(\frac{M_{1}(0)^{2}}{c_{1}}+\frac{M_{2}(0)^{2}}{c_{2}}+\frac{M_{3}(0)^{2}\left(M_{1}(0)^{2} / c_{1}+M_{2}(0)^{2} / c_{2}\right)}{M_{1}(0)^{2}+M_{2}(0)^{2}}\right) \\
& M=\left(M_{1}(0)^{2}+M_{2}(0)^{2}+M_{3}(0)^{2}\right)
\end{aligned}
$$

then we optimise the parameters to minimize the function:

$$
\min _{M_{1}(0), M_{2}(0), M_{3}(0), c_{1}>0, c_{2}>0, a_{1}, a_{2} \in \mathbb{R}}\left(\left\|x^{\prime}-x_{d}\right\|_{\mathbb{R}^{3}}+\alpha \mathbf{u}_{a}\right)
$$

where $x^{\prime}=R(0)^{-1} x(T)$ where $x$ is defined in (28) and evaluated at the final fixed time $T$ and $x_{d}$ is the final desired pointing direction at time $T$. Also note that $a_{1}, a_{2}$ are included in the optimisation simply to ensure the correct choice of the signs $s_{1}=a_{1} /\left|a_{1}\right|, s_{2}=a_{2} /\left|a_{2}\right|$. In terms of practical implementation it may be computationally more efficient to discretise $\mathbf{u}_{a}=\int_{0}^{T}\|\mathbf{u}(t)\|_{\mathbb{R}^{3}} d t$. For example one simple way of doing this is to write:

$$
\int_{0}^{T}\|\mathbf{u}(t)\|_{\mathbb{R}^{3}} d t \approx \frac{T}{n}\left(\frac{\|\mathbf{u}(0)\|_{\mathbb{R}^{3}}+\|\mathbf{u}(T)\|_{\mathbb{R}^{3}}}{2}+\sum_{k=1}^{n-1}\left\|\mathbf{u}\left(k \frac{T}{n}\right)\right\|_{\mathbb{R}^{3}}\right)
$$

where in our simple example we set $T=100, n=5$ and $\alpha=1$. The cost function (34) is minimised using the random search algorithm in Mathematica and returned the values $M_{1}(0)=0.0000352301, M_{2}(0)=-0.0404792, M_{3}(0)=$ $0.0415471, c_{1}=1.41766, c_{2}=2.05914$ with $s_{1}, s_{2}=1$. This corresponds to the motion and torque profile illustrated in Figures 1 and 2. As can be seen the planned motions can be performed well within the range of the maximum torque of current nano-reaction wheels. The motions could be further optimised by considering the final time $T$ as a free parameter and by tuning the weight in cost function to suit the desired tolerances of the application. Note that this is only a preliminary investigation and there are many parts

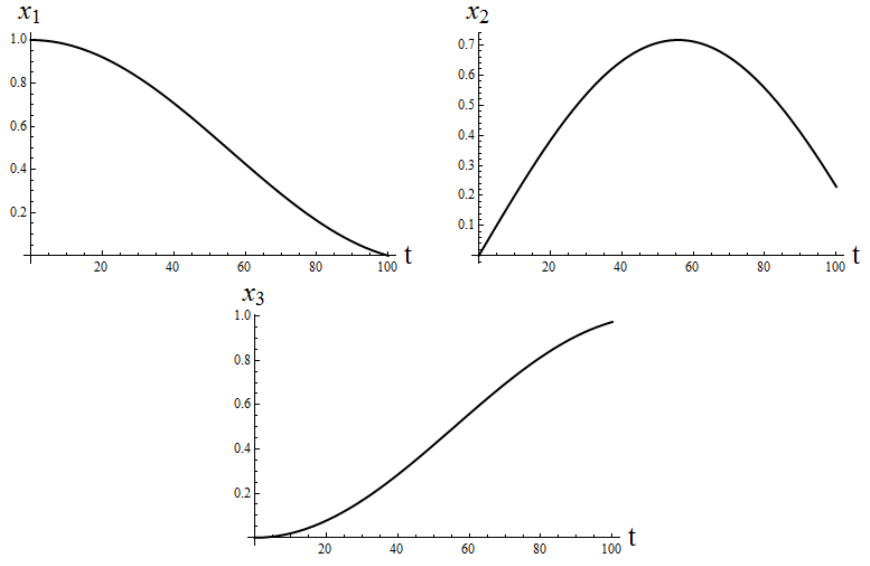

Fig. 1. Planned pointing motion $x=\left[x_{1}, x_{2}, x_{3}\right]^{T}$ over time $t \in[0, T]$ (secs) between $x_{0}=[1,0,0]^{T}$ and $x_{f}=\left[\begin{array}{lll}0 & 0.22942 & 0.97333\end{array}\right]^{T}$
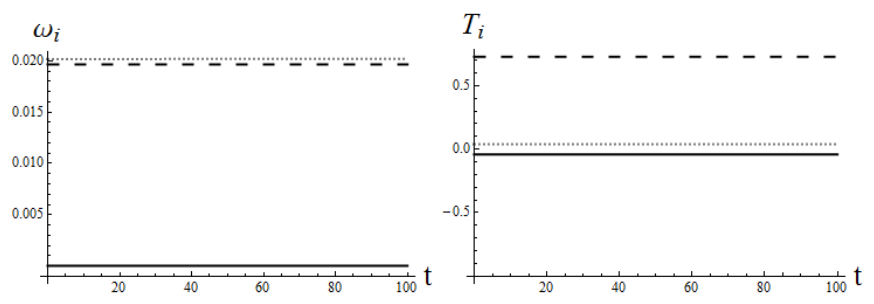

Fig. 2. The second figure illustrates the angular velocities $\omega_{1}$ (Dashed line), $\omega_{2}$ (Continuous line), $\omega_{3}$ (Dotted line) (rads/sec). The second figure illustrates the ideal torques $T_{1}$ (Dashed line), $T_{2}$ (Dotted line), $T_{3}$ (Continuous line) $\left(\times 10^{-5} \mathrm{Nm}\right)$

of the algorithm that can be improved. For example, in this paper only a simple random search was used to optimise the parameters of the cost function and an investigation into the most appropriate optimiser for this application is required. Furthermore, the angular velocities are not restto-rest motions and therefore not ideal for performing such motions. However, the angular velocities are very small and their boundaries at $t=0$ and $t=T$ can be matched using an appropriate tracking controller. To illustrate this we use a tracking controller in a simulation of spacecraft attitude. We assume that the spacecraft is equipped with simple reaction wheels. The wheel data is based on the Sinclair Interplanetary pico-satellite reaction wheels (with a maximum torque of $1 \times 10^{-3} \mathrm{Nm}$. In the environmental model the spacecraft is subjected to disturbance torques due to gravity gradient, solar radiation pressure and air drag, and the residual dipole of the spacecraft. The magnetic field is modelled using a simple dipole model, rotated to mirror the offset between the geographic and geomagnetic poles. The spacecraft is considered to be on a $600 \mathrm{~km}$ altitude circular orbit beginning at the vernal equinox position. The tracking controller used is of the form:

$$
\mathbf{u}=-K_{1} \omega_{e}-K_{2} y
$$

where $y$ is the vector form of $\hat{y}$ defined in (31) and where $K_{1}, K_{2}$ are $3 \times 3$ gain matrices that are manually tuned. In order to perform a rest-to-rest motion using references that 
are not rest-to-rest (but where the initial and final angular velocities are small) the following control logic is used to define the desired motion $R_{d}, \omega_{d}$ that is tracked in practise:

$$
\begin{aligned}
& \text { For } t=\left[0, T_{L}-\tau\right) \quad\left\{\begin{array}{c}
\omega_{d}=\omega_{\text {ref }} \\
R_{d}=R_{\text {ref }}
\end{array}\right. \\
& \text { For } t=\left[T_{L}-\tau, T_{L}\right] \quad\left\{\begin{array}{c}
\omega_{d}=0 \\
R_{d}=R_{\text {ref }}(T)
\end{array}\right.
\end{aligned}
$$

where $T_{L}=T+\tau$ where $T_{L}=120$ is the total length of the motion including the time $\tau=20$ required to stabilise the final pointing motion and the time, $T=100$, of the planned motion where $\omega_{r e f}$ and $R_{r e f}$ define the reference motion generated by the motion planner. Using this control the reference rotation was tracked very closely. A figure of the actual rotation compared to the reference rotation is not included as the two motions are visually inseparable. However, the real angular velocities are required to be restto-rest and are illustrated in Figure 3. As the reference motion
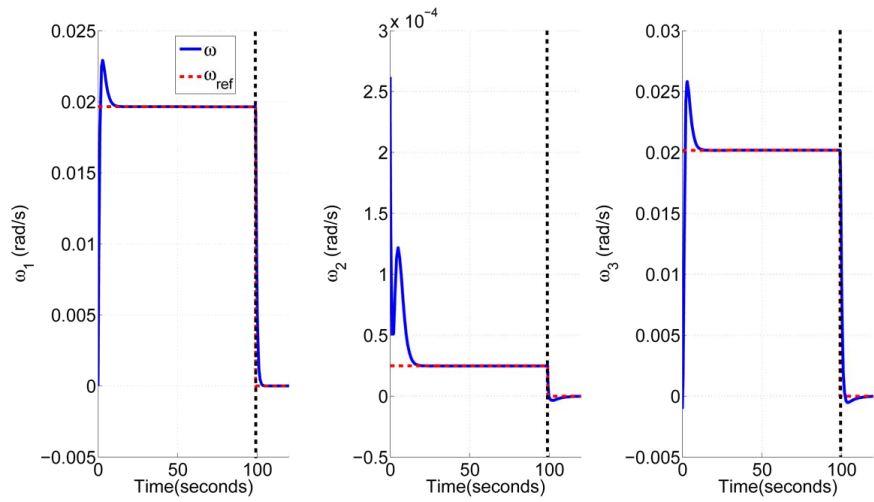

Fig. 3. Angular velocity profile for a rest-to-rest motion (rads/sec). The vertical dashed line indicates the switch in reference.

is not rest-to-rest a burst of torque is required at the beginning and end of the motion. This can be seen in the time history of the control torques during the maneuver in Figure 4. It can be

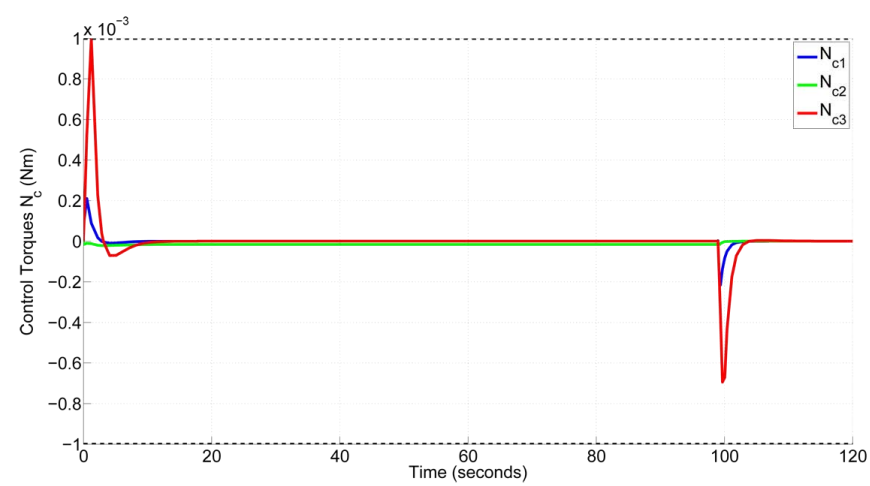

Fig. 4. Real torque (Nm) $N_{c}=\left[N_{c 1}, N_{c 2}, N_{c 3}\right]^{T}$ over time $t \in[0, T]$ (secs). The dashed horizontal lines represent torque limits.

seen from Figure 4 that even in the presence of environmental perturbations, actuator constraints and the requirement of matching the zero angular velocities at the end-points that the motion can be completed within the feasible range of nano-spacecraft reaction wheels.

\section{CONCLUSION}

A procedure for generating reference motions for attitude control has been presented. The method is based on pruning the number of feasible motions to define a class of motions of hyperbolic type. Amongst this subset of analytically defined motions the accumulated torque and final pointing error can be minimised by optimising a small number of free parameters. Future work is required to evaluate the effectiveness of the motion planner used with a tracking controller on the overall effectiveness of the ADCS. In addition a comparison with other motion planning methods in terms of torque requirement, pointing accuracy and computational expense is required. However, it must be noted that the parameter optimisation problem in this paper is restricted to matching boundary conditions on the orientation and not on the velocities. Future work will investigate methods to re-parameterise time in these analytic solutions so that pre-defined boundary conditions on the velocity can be matched. Furthermore, future work will investigate the most appropriate numerical optimiser and to investigate the use of time as a free parameter.

\section{ACKNOWLEDGMENTS}

This work has been supported by the Marie Curie fellowship PITN-GA-2011-289240 AstroNet-II and the Technology Strategy Board: Space for Growth Programme.

\section{REFERENCES}

[1] Wie, B., 'Space vehicle dynamics and control'. AIAA Education, 1998.

[2] Chaturvedi, N., Sanyal, A., McClamroch, N.,'Rigid-body attitude control: using rotation matrices for continuous, singularity-free control laws'.IEEE control systems magazine, June 2011.

[3] Kang, W., Bedrossian, N., 'Pseudospectral optimal control theory makes debut flight, saves NASA $\$ 1 \mathrm{M}$ in under three hours'.SIAM news, vol. 40, No. 7,2007.

[4] Caubet, A., Biggs, J., 'An Optimal and Efficient Attitude Motion Planner Using a Polynomial Shape-Based Method for Large Slew Maneuvers' AIAA Guidance, Navigation, and Control Conference, Boston, Massachusetts, USA, August 2013.

[5] Biggs, J., Horri, N.,'Optimal geometric motion planning for a spinstabilized spacecraft'. Systems \& Control Letters, vol.61, issue 4. pp. 609-616, 2012.

[6] Pagnozzi, D., Maclean, C., Biggs, J "A new approach to the solution of free rigid body motion for attitude manoeuvers". In: European Control Conference, ECC 2013, 2013-07-17 - 2013-07-19, Zurich.July 2013.

[7] Maclean, C., Pagnozzi, D., Biggs, J "Computationally light attitude controls for resource limited nano-spacecraft.". Proceedings of the 62nd International Astronautical Congress, Cape Town, , South Africa, 2011.

[8] Jurdjevic, V., 'Geometric Control Theory'. Advanced Studies in Mathematics, Cambridge University Press, 52, 1997.

[9] Spindler, K., 'Optimal attitude control of a rigid body'. Applied mathematics and optimization, No. 34, pp. 79-90, 1996.

[10] Biggs, J.D. Singularities of optimal attitude motions. In: 18th IFAC Symposium on Automatic Control in Aerospace, ACA 2010, 2010-0906 - 2010-09-10, Nara, Japan.2010

[11] Biggs, J., 'Quadratic Hamiltonians on non-Euclidean spaces of arbitrary constant curvature.' European Control Conference, Zurich, July 2013.

[12] M. Abramowitz, I.A. Stegun, 'Handbook of Mathematical Functions', Dover, New York, 1972.

[13] Iserles, A., Munthe-Kaas, H, Norsett, S., Zanna, A.,'Lie-group methods'. Acta Numerica, Springer, 9, pp. 215-365, 2000. 